\section{(A) Check for updates}

Cite this: Food Funct., 2021, 12 12162

DOI: $10.1039 / \mathrm{d} 1 \mathrm{fo} 90072 \mathrm{~b}$

rsc.li/food-function

\title{
Correction: Bioactive extracts from brewer's spent grain
}

\author{
Teresa Bonifácio-Lopes, (DD ${ }^{a, b}$ Ana A. Vilas Boas, (D) a Ezequiel R. Coscueta, (D) a \\ Eduardo M. Costa, (D) ${ }^{a}$ Sara Silva, (D) a Débora Campos, (D) ${ }^{a}$ José A. Teixeira (D) ${ }^{b}$ and \\ Manuela Pintado (iD *a
}

Correction for 'Bioactive extracts from brewer's spent grain' by Teresa Bonifácio-Lopes et al., Food Funct., 2020, 11, 8963-8977, DOI: 10.1039/DOFO01426E.

The authors regret that an incorrect version of Table 2 was included in the original article. The correct version of Table 2 is presented below.

Table 2 Total phenolic content and antioxidant capacity of the BSG extracts (mean \pm S.D)

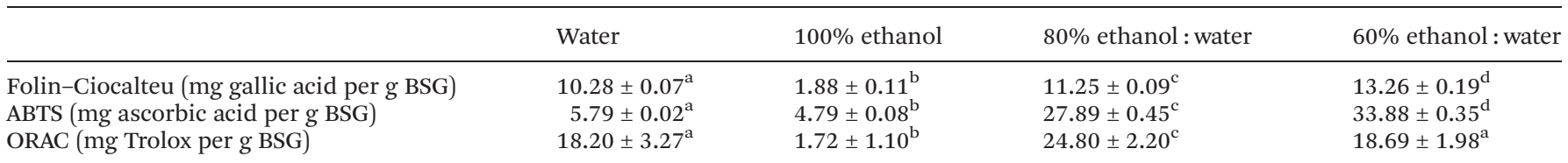

The data is given in mean \pm S.D (standard deviation). For each column, equals letters it means that the difference is not significant at $p>0.005$.

"Consequently, sections of the text in the manuscript should be adjusted according to this change, and these are detailed below.

The paragraph on page 8968 beginning "As it can be seen in Table 2..." should be correctly given as follows:

"As it can be seen in Table 2, the content of total phenolics (TPC) varied from $1.83 \mathrm{mg}$ gallic acid per $\mathrm{g}$ BSG, when ethanol was used, to $13.26 \mathrm{mg}$ gallic acid per $\mathrm{g}$ BSG for the $60 \%$ ethanol : water extract, which represents a 0.9 fold increase in the extraction yield. It is worth highlighting that the highest amount of TPC obtained in the present work (13.26 mg gallic acid per $\mathrm{g}$ BSG) was higher than the values previously reported by McCarthy et $a .^{41}$ (1.26-4.53 mg gallic acid per $\mathrm{g}$ BSG) by alkaline hydrolysis. It is also possible to see that the extraction with $60 \%$ ethanol : water was more efficient than the one performed with $100 \%$ ethanol. According to Spigno et $a l .{ }^{42}$ the addition of water to organic solvents leads to the increase of the polarity of medium, which facilitates phenolic compound extraction. The results obtained in the present work (Table 2) also showed that the extraction using only water was not as efficient as when using ethanolic mixtures as solvents, since phenolic compounds have higher affinity for less polar medium, such as ethanol. ${ }^{43}$ "

The sentence beginning " This value is much lower compared...” should be correctly given as "This value is much lower compared to that for the $60 \%$ ethanolic extract, since it showed ca.7 times less activity in the case of the ABTS method and ca. 10 times less than in the case of the ORAC method."

The sentence beginning "The highest values of ABTS..." should be correctly given as "The highest values of ABTS assay were obtained with $60 \%$ ethanolic extract, which was 5 times higher than the water extract and very close to the $80 \%$ ethanolic extract."”

The Royal Society of Chemistry apologises for these errors and any consequent inconvenience to authors and readers.

${ }^{a}$ Universidade Católica Portuguesa, CBQF - Centro de Biotecnologia e Química Fina - Laboratório Associado, Escola Superior de Biotecnologia, Rua Diogo Botelho 1327, 4169-005 Porto, Portugal. E-mail: mpintado@porto.ucp.pt

${ }^{b}$ CEB - Centre of Biological Engineering, University of Minho, 4710-057 Braga, Portugal 